\title{
Metasurface virtual absorbers: unveiling operative conditions through equivalent lumped circuit model
}

\author{
Angelica Viola Marini ${ }^{*}$, Davide Ramaccia, Alessandro Toscano, and Filiberto Bilotti \\ Department of Engineering, ROMA TRE University, Via Vito Volterra 62, Rome 00146, Italy
}

Received: 29 October 2020 / Accepted: 20 December 2020

\begin{abstract}
Virtual absorption concept has been recently introduced as a new phenomenon observed in electromagnetics and optics consisting of theoretically unlimited accumulation of energy within a finite volume of material without dissipation. The anomalous behaviour is achieved by engaging the complex zero scattering eigenmodes of the virtual absorbing system by illuminating it with a proper complex frequency $\omega=\omega_{r}+j \omega_{i}$, whose value is strictly determined by the system characteristics. In this paper, we investigate on the position of the zero-pole scattering pairs in the complex frequency plane as a function of the input impedance of the metasurface-based lossless virtual absorber. We analytically derive the conditions under which a properly modulated monochromatic plane wave can be virtually absorbed by the system and stored within its volume. The analysis is developed by modelling the propagation of a normally impinging plane wave through its equivalent transmission line model terminated in an arbitrary reactive load, which in turn models the input impedance of the metasurface-based system under consideration. The study allows to determine $a$ priori whether the metasurface-based system can support the virtual absorption or not by evaluating the time-constant from its equivalent circuit.
\end{abstract}

Keywords: Virtual perfect matching / time-varying signal / complex frequency / virtual absorption

\section{Introduction}

The localization and confinement of electromagnetic energy in a finite volume without dissipation has been extensively studied by the scientific community in the last years, by exploiting the anomalous interaction between light and artificial metastructures [1-19]. In this framework, virtual absorption concept [20-24] represents one of the most appealing technique for storing and releasing electromagnetic energy, due to passive and simple structures that can support the desired energy accumulation. Indeed, originally the virtual absorption has been achieved by illuminating a lossless dielectric slab of finite thickness with two perfectly synchronized signals at its opposite sides, demonstrating the possibility to achieve zero total scattering and energy accumulation when the illuminating signals have a proper complex temporal frequency $\omega=\omega_{r}+j \omega_{i}[20]$. This special temporal excitation is fundamental for engaging the complex zero scattering eigenmodes of the system and enabling its anomalous electromagnetic behaviour of zero scattering and energy storing.

\footnotetext{
* e-mail: angelicaviola.marini@uniroma3.it
}

More recently, to overcome the strictly requirements of pure lossless materials and perfect coherent illumination of the structure, we proposed a metasurface-based virtual absorber $[25,26]$, able to store energy from an impinging plane wave under arbitrary illumination condition, and without the use of dielectric materials. As shown in Figure 1a, the proposed structure consists of a 1D cavity filled by vacuum and bounded by a reflector and a metasurface. The metasurface is designed to be penetrable by the external illuminating field, allowing the cavity to interact with the external environment. In [25], exploiting the transmission line equivalence in Figure 1b, we analytically derived the required complex frequency of the illuminating plane wave as a function of the surface reactance $X_{m t s}$ of the metasurface, the electrical size of the cavity, polarization and incidence angle of the illuminating wave. In [25] we also reported an effective design guideline of a metasurface-based virtual absorber for a given frequency, noticing that the metasurface reactance value was strongly linked with the electrical size of the cavity (Eq. (5) in [25]). This suggests that the virtual absorption phenomenon does not occur for any pair of metasurface and cavity impedances.

The aim of this paper is to identify the conditions in terms of equivalent lumped impedances modelling the 


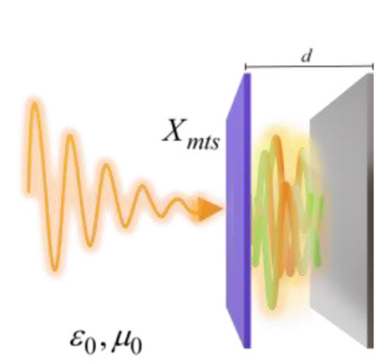

(a)

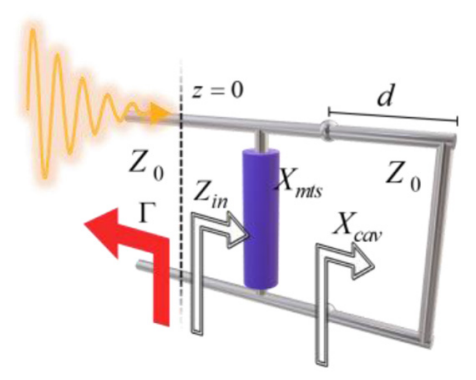

(b)

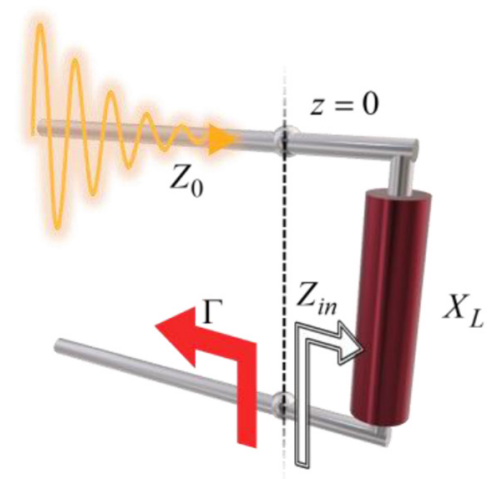

(c)

Fig. 1. Schematic representation of (a) a virtual metasurface absorber, (b) its transmission line model, and (c) circuital equivalent transmission line with lumped elements.

metasurface and cavity that enable virtual absorption. Therefore, the entire analysis is reduced to the circuital response of the transmission line model shown in Figure 1b. The metasurface reactance $X_{m t s}$ and input reactance $X_{c a v}$ of the short-circuited stub modelling the metal-backed cavity can assume independently capacitive and inductive reactive values, according to the metasurface layout and electrical dimension of the cavity. Their overall response can be synthesized through the reactive load $X_{L}$ shown in Figure 1c. Such a representation significantly simplifies the analysis by reducing the problem of virtual absorption to a very well-established concept in microwave circuit, that is perfect impedance matching [27]. Indeed, when the transmission line in Figure 1c is matched under complex excitation, the corresponding metasurface-bounded open cavity in Figure 1a is virtually absorbing the impinging electromagnetic wave, allowing us to easily identify the operative bounds for the proposed system. By exploring the scattering zero-pole pairs and their position in the complex frequency plane as a function of such reactances, we analytically derive the conditions under which the system can support virtual absorption and the corresponding operative bounds.

The paper is organized as follows. In Section 2 we introduce the analysis method by focusing our attention on the response from the load in terms of reflection coefficient when an excitation signal with complex frequency is propagating along the feeding transmission line. Here, we investigate the two possible configurations for the input reactance $X_{L}$ given by the parallel connection of surface reactance $X_{m t s}$ and input reactance $X_{c a v}$ of the shortcircuited stub modelling the metal-backed cavity: (i) $X_{m t s}$ and $X_{\text {cav }}$ have the same equivalent electrical response, that is, both capacitive or both inductive; and (ii) $X_{m t s}$ and $X_{c a v}$ have opposite equivalent electrical responses, that is, one capacitive and one inductive. In Section 3 we verify the perfect matching of the reactive load in the two configurations identified in Section 2 by a proper set of numerical simulations in ADS [28]. We demonstrate that if the lumped-element equivalent modelling the metasurface virtual absorber exhibits the proper values, the virtual absorption can be enabled. Finally, in Section 4 some conclusions are drawn.

\section{Operative conditions of metasurface virtual absorber through TL model}

Let us consider an arbitrary metasurface virtual absorber as shown in Figure 1a. Under plane wave excitation from free-space, the propagation and interaction with the virtual absorber can be modelled as shown in Figure 1b: a lossless transmission line with characteristic impedance modelling the surrounding vacuum $Z_{0}=\sqrt{\mu_{0} / \varepsilon_{0}} \simeq 377 \Omega$ is loaded by a parallel connection between the reactances $X_{m t s}(\omega)$ and $X_{\text {cav }}(\omega)$. The response of the system can be derived by evaluating the amplitude of the reflection coefficient at the terminals of the Thevenin impedance $j X_{L}(\omega)=j X_{m t s}(\omega) \|$ $j X_{\text {cav }}(\omega)$, where "||" denotes parallel connection:

$$
|\Gamma(\omega)|=\left|\frac{Z_{\text {in }}-Z_{0}}{Z_{\text {in }}+Z_{0}}\right|=\left|\frac{j X_{L}(\omega)-Z_{0}}{j X_{L}(\omega)+Z_{0}}\right| .
$$

The overall input impedance $Z_{i n}$ is a frequency dependent quantity and is purely imaginary. Being $Z_{0}$ a real valued impedance, the system turns out to be always mismatched, since $j X_{L} \neq Z_{0}$ over the whole real frequency spectrum. To achieve virtual absorption, that is, perfect impedance matching from the circuital point-of-view, the imaginary quantity $j X_{L}(\omega)$ should be converted into a real one and equated to the real characteristic impedance $Z_{0}$. This can be done by introducing a complex value of the excitation frequency $\omega$, that is, $\omega=\omega_{r}+j \omega_{i}[25]$. A complex frequency excitation is a harmonic signal of frequency $\omega_{r}$, whose amplitude temporally varies following an exponential growing or decaying profile proportional to $\exp \left(\omega_{i} t\right)$. Under such particular excitation condition, the reflection coefficient in equation (1) presents some scattering singularities in the complex frequency plane. The singularities consist of zero-pole pairs as complex conjugated values. Each pair occurs periodically over the real frequency axis, as shown in $[20,21]$, due to the fact that the cavity exhibits the same input reactance for different cavity sizes [29].

On the contrary, using lumped-elements representation (Fig. 1c) where $X_{m t s}, X_{c a v}$ are modelled through a lumped inductor or capacitor, the equivalent reflection coefficient complex frequency plane will lose the periodic behaviour of scattering singularities, but will preserve the information on the feasibility of triggering the virtual absorption phenomenon according to the values of reactances $X_{m t s}$ and $X_{c a v}$. 


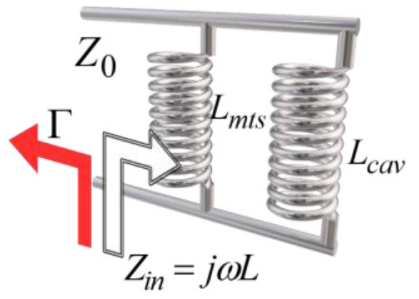

(a)

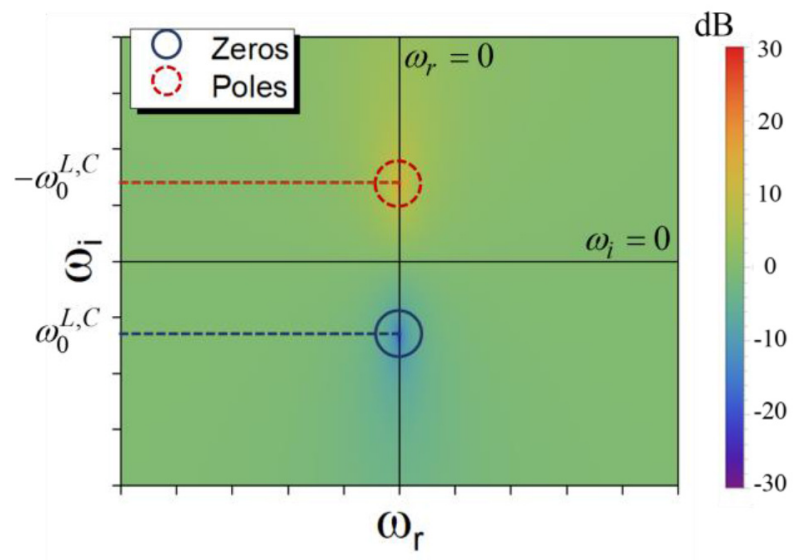

(c)

Fig. 2. Parallel connection of two reactances with the same electrical behaviour (a) both inductive and (b) both capacitive. (c) Amplitude of the reflection coefficient of the loaded transmission line in the complex frequency plane.

In the following subsections, we consider two relevant cases consisting of two different combinations for the metasurface and cavity reactances: in the former, the two reactances show the same electrical behaviour $\left(X_{m t s} \cdot X_{c a v}>0\right)$, whereas, in the latter, the opposite one $\left(X_{m t s} \cdot X_{c a v}<0\right)$.

\subsection{Metasurface and cavity with same electrical behaviour}

Let us now consider the case where $X_{m t s}$ and $X_{\text {cav }}$ have the same equivalent electrical responses, that is, both capacitive or both inductive. In Figures $2 \mathrm{a}$ and $2 \mathrm{~b}$, we report the transmission line when the load consists of a parallel connection of two inductors or two capacitors, and realises an input impedance $Z_{i n}=j X_{L}(\omega)$, where $X_{L}=\omega L$ and $X_{L}=-1 / \omega C$, respectively, with $L$ and $C$ being the total inductance and capacitance measured at the load terminals.

The reflection coefficients for the two considered scenarios can be easily written as:

$$
\Gamma^{L}(\omega)=\frac{j \omega \tau-1}{j \omega \tau+1}, \Gamma^{C}(\omega)=-\Gamma^{L}(\omega)
$$

where $\tau$ assumes the values $\tau^{L}=L / Z_{0}$ and $\tau^{C}=Z_{0} C$ in case of inductive and capacitive load, respectively.
Substituting the complex frequency $\omega=\omega_{r}+j \omega_{i}$ in equation (2), the amplitude of the reflection coefficients can be plotted in the complex frequency plane, as shown in Figure 2c.

It is interesting to note that, regardless the reactive load type, the amplitude of the reflection coefficient exhibits two singularities: a pole is in the positive halfspace of the imaginary frequency, whereas a zero is in the negative half-space. Both of them exist for the same real frequency $\omega_{r}=0$ and are complex conjugate quantities. From the physical point of view, the pole represents the case when the load is reflecting more energy than the impinging one. In passive systems, this is as a special scattering condition for which the scattered field decays slower than the excitation field, giving rise to a virtual gain effect, as shown in [30]. On the contrary, the zero corresponds to a zero-reflection condition, which behaves as an indefinite accumulator for the illuminating signal, without dissipating its energy but rather storing it within the reactive load. The zero-scattering condition is kept if the complex frequency is such to approach the zero of the reflection coefficients. As soon as the frequency of the signal changes, the reflection coefficient assumes a non-zero value and energy leaks out from the reactive load.

By forcing equation (2) to zero, we obtain that the reflection coefficient vanishes at the complex frequencies:

$$
\omega_{0}^{L, C}=\omega_{r}+j \omega_{i}=0-j \tau^{-1}, \text { with } \tau=\tau^{L, C} .
$$

The complex frequencies $\omega_{0}^{L, C}$ are purely imaginary $\left(\omega_{r}=0\right)$ in equation (3). Being the zero and the pole a complex conjugate pair, they have the same imaginary frequency amplitude $\left|\omega_{i}\right|$ (Fig. 2c). The signal, then, is not oscillating in time, but simply exponentially growing. The steepness in time of the applied signal is given by the exponential factor $\exp \left(-\omega_{i} t\right)$. This is fully consistent with circuit theory where the charging and discharging behaviours of $R C$ and $R L$ circuits are described by the same exponential curve with a factor $\exp (-t / \tau)$ for voltage and current, respectively.

To conclude, when metasurface and cavity exhibit the same equivalent electrical behaviour, the metasurfacebased virtual absorbing condition cannot be achieved, since the propagation of the exciting field requires a non-zero real frequency to propagate.

\subsection{Metasurface and cavity with opposite electrical behaviour}

Let us now consider the case where $X_{m t s}$ and $X_{c a v}$ have opposite equivalent electrical responses, that is, $X_{m t s} \cdot X_{c a v}<0$. Therefore, the lossless transmission line is loaded by a reactive network modelled as parallel connection of an inductor $L$ and capacitor $C$ (Fig. 3a), leading to a load reactance:

$$
j X_{L}=j \frac{\omega L}{1-\omega^{2} L C},
$$




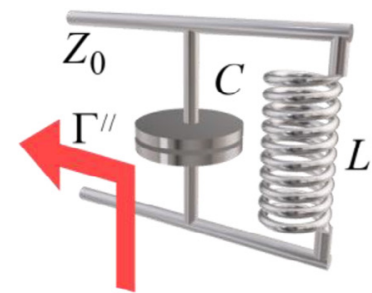

(a)
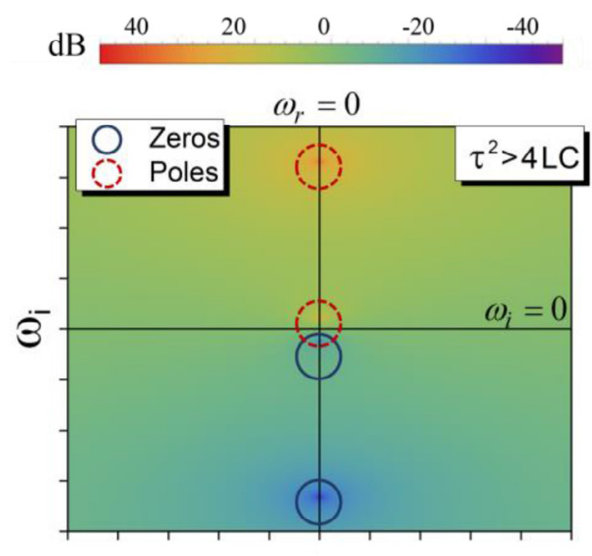

(b)

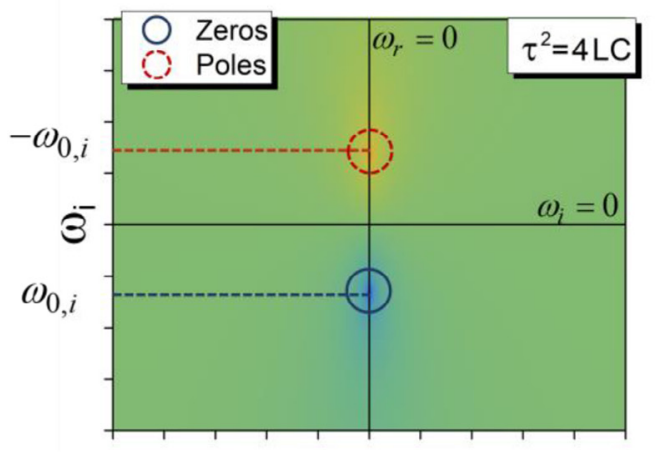

(c)

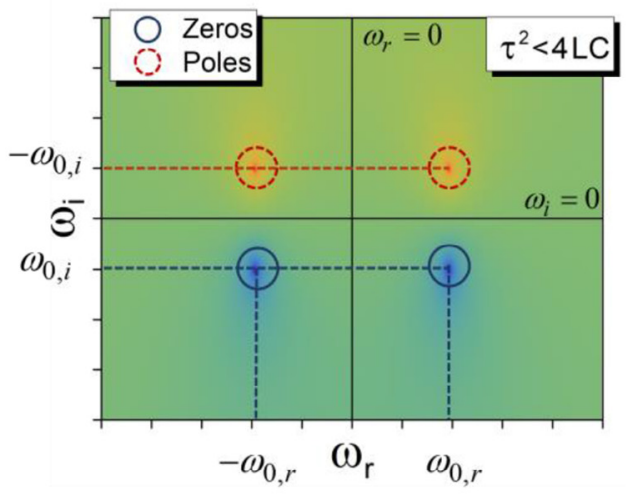

(d)

Fig. 3. Quality behaviour of the reflection coefficient amplitude $\Gamma$ in the complex plane showing zeros in lower half-plane and poles in higher half-plane. Respectively zeros are, for a (a) parallel connection of an $L C$ load case study, (b) purely imaginary when $\tau^{2}>4 L C,(\mathrm{c})$ coincident purely imaginary when $\tau^{2}=4 L C$, (d) complex when $\tau^{2}<4 L C$.
Substituting equation (4) into equation (1), we obtain:

$$
\Gamma(\omega, \tau)=-\frac{\left(\omega^{2} L C-1\right)+j \omega \tau}{\left(\omega^{2} L C-1\right)-j \omega \tau}
$$

where $\tau=L / Z_{0}$ is the circuit time constant defined according to circuit theory. By forcing equation (5) to vanish, we can obtain the following perfect matched complex frequencies:

$$
\omega_{0}=\frac{1}{j}\left(\frac{\tau \pm \sqrt{\tau^{2}-4 L C}}{2 L C}\right) .
$$

It is worth noticing that, at a first glance, equation (6) seems to identify pure imaginary frequencies, as in the case reported in Section 2.1. However, this is true only if the argument of the square root in equation (6) is positive. Conversely, for negative arguments, the square root returns an imaginary quantity, that allows achieving a complex frequency with non-zero real part, that is, harmonic signals at frequency $\omega_{r}$ with temporal varying envelope, growing or decaying, according to the frequency $\omega_{i}$. In this case, two distinct regions can be identified, separated by the special condition for which the argument of the square root is zero, that is, $\tau^{2}=4 L C$. If $\tau^{2} \geq 4 L C$, the complex frequencies in equation (6) are purely imaginary, as for the case in Section 2.1, whereas, if $\tau^{2}<4 L C$, the angular frequency can assume complex values.

It is interesting now to plot the amplitude of the reflection coefficient in equation (5) for the three distinct cases: (i) $\tau^{2}>4 L C$, (ii) $\tau^{2}=4 L C$, and (iii) $\tau^{2}<4 L C$, as a function of the complex frequency. The plots are reported in Figures 3b-3d. The reflection coefficient exhibits some singularities in the spectrum, that are located differently in each considered case:

(i) $\left(\tau^{2}>4 L C-\right.$ Fig. $\left.3 \mathrm{~b}\right)$ the reflection coefficient has two pairs of singularities, zeros and poles are symmetrically distributed and lying along the imaginary axis. All singularities have zero real frequency.

(ii) $\left(\tau^{2}=4 L C-\right.$ Fig. $\left.3 \mathrm{c}\right)$ the two poles and the two zeros degenerate, allowing only one zero to be engaged. Again, the singularities are located along the axis $\omega_{r}=0$, meaning that the excitation signal is not oscillating with a specific real frequency as desired for achieving matching in microwave networks.

(iii) $\left(\tau^{2}<4 L C-\right.$ Fig. $\left.3 \mathrm{~d}\right)$, the reflection coefficient exhibits two zeros for complex frequencies with a non-zero real part.

This last case is of fundamental importance for our analysis because it demonstrates that it is theoretically possible to achieve zero reflection from a metasurfacebased virtual absorber, when the metasurface and the cavity show an opposite electrical behaviour in the load reactance (i.e., parallel connection of an inductor and capacitor). This occurs under monochromatic excitation with a signal at frequency $\omega_{r}$, provided that the amplitude of the wave follows the profile $\exp \left(-\omega_{i} t\right)$. It is worth 
noting that Figure 3d reports two possible zeros symmetrically distributed with respect to the imaginary axis, but only the zero for positive real frequency can be excited. Hence the left pair of pole-zero can be neglected.

\section{Results and verification}

This section is dedicated to the validation of operative conditions for an arbitrary metasurface-based virtual absorber as identified in Section 2. In the following, a metasurface with $X_{m t s}=100 \Omega$ at $f_{0}=692 \mathrm{MHz}$ is considered, which will be modelled through an inductor $L_{m t s}=X_{m t s} /\left(2 \pi f_{0}\right)=23 \mathrm{nH}$. The metasurface value is kept in the different configurations, whereas the cavity is time-by-time selected to fit with one of the specific cases discussed in Section 2. The simulations are performed by loading a scattering port with an internal impedance of $377 \Omega$ with the different equivalent lumped elements modelling the metasurface and cavity. The incident and reflected signals are recorded directly at the scattering port terminals.

Let us start considering an inductive cavity with electrical thickness $0.02 \lambda_{0}$, showing an input reactance $X_{c a v}=47.6 \Omega$, which can be modelled through an inductor $L_{\text {cav }}=X_{\text {cav }} /\left(2 \pi f_{0}\right)=11 \mathrm{nH}$. According to equation (3) the inductance $L=L_{m t s} \| L_{\text {cav }}$ exhibits zero reflections at the imaginary frequency $\omega_{1}=\left(0-j 5.1 \times 10^{10}\right) \mathrm{rad} / \mathrm{s}$, that is, a non-oscillating time-varying signal whose amplitude grows in time, as shown in Figure 4a (orange line). As expected, as long as the voltage signal is applied to the circuit, no reflections occur till the kick-off time $t_{0}$. Being the entire circuit passive, energy cannot be dissipated, allowing only energy storing in the reactive load. As soon as the signal stops, the zero-reflection condition is not satisfied anymore, forcing the reactive load to release the stored energy, as shown in Figure 4a (green line). From the electrical point of view, this corresponds to the charging and discharging behaviours of an $R L$ circuit, where the resistive element controlling the damping factor is the freespace impedance $Z_{0}$. However, this case cannot be implemented in a realistic metasurface virtual absorber, due to the impossibility to excite it with a propagating monochromatic electromagnetic field.

Let us now consider a capacitive cavity with electrical thickness $0.46 \lambda_{0}$, showing an input reactance $X_{\text {cav }}=-94 \Omega$, which can be modelled through a capacitor $C_{\text {cav }}=1 /\left(2 \pi f_{0} X_{\text {cav }}\right)=2.45 \mathrm{pF}$. The cavity size has been selected in order to satisfy the condition $\tau^{2}<4 L C$, which enables perfect matching also when the excitation is oscillating with a real frequency $\omega_{r} \neq 0$. Indeed, according to equation (6), the zero of the reflection coefficient is achieved at the frequency $\omega_{2}=\left(2 \pi f_{0}-j 5.4 \times 10^{8}\right)$, that is, a monochromatic signal at frequency $f_{0}$, with a time-varying growing envelop following the profile $\exp \left(5.4 \times 10^{8} t\right)$, as shown in Figure $4 \mathrm{~b}$ (orange line). Again, the voltage signal is virtually absorbed by the reactive load as for the previous case, storing the energy in the LC reactive load, and controlling the releasing through the kick-off instant of time. It is worth mentioning that, in this case, the system has its realistic free-space counterpart, being the exciting signal oscillating in time and supporting propagation as electromagnetic wave [25].

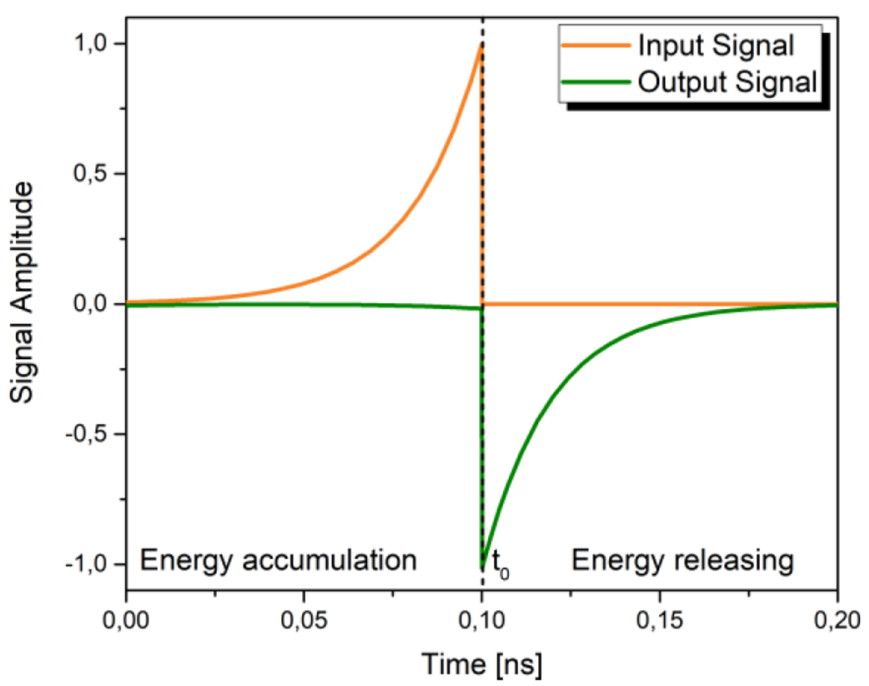

(a)

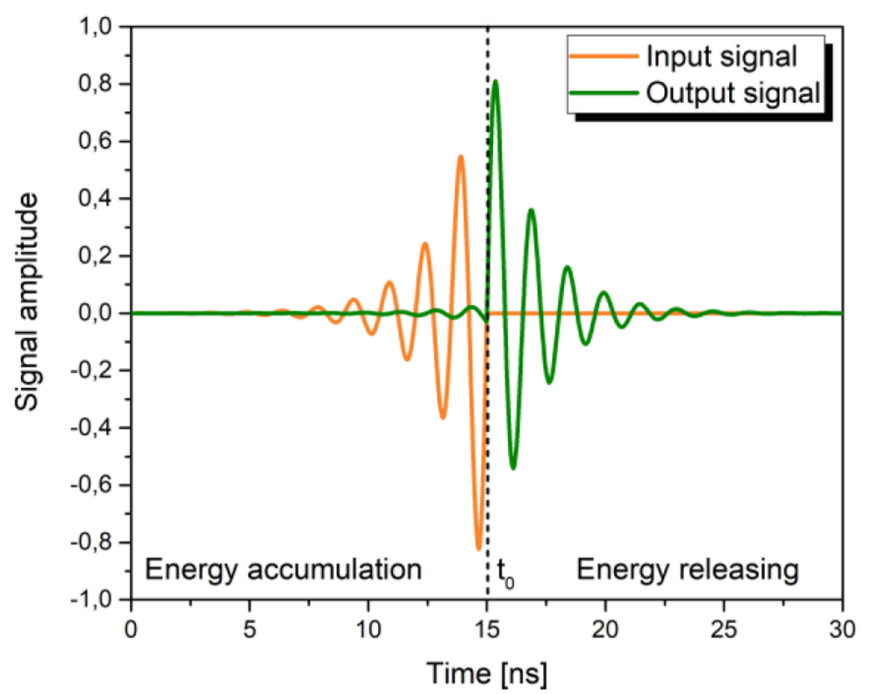

(b)

Fig. 4. Amplitude of the incident and released signals of the reactive load for parallel connection of $L_{m t s}=23 \mathrm{nH}$ and (a) $L_{c a v}=11 \mathrm{nH}$ and (b) $C_{c a v}=2.45 \mathrm{pF}$.

\section{Conclusion}

In this paper, we have explored the analytical operation limits to get virtual absorption for a metasurface-based absorber. By investigating on its lumped circuit equivalent loads, we have also found the conditions enabling the perfect matching of purely reactive loads by exploiting time-varying complex signals for metasurface and cavity reactances showing the same $\left(X_{m t s} \cdot X_{c a v}>0\right)$ and the opposite $\left(X_{m t s}\right.$. $X_{\text {cav }}<0$ ) electrical behaviour. For these cases, we have theoretically derived the fundamental limits of the impedance matching concept based on time-modulated excitation signals, in terms of load configurations, and ranges of values for load inductance and capacitance for which the perfect matching can be achieved. 


\section{References}

1. F. Monticone, H.M. Doeleman, W. Den Hollander, A.F Koenderink, A. Alù, Trapping light in plain sight: embedded photonic eigenstates in zero-index metamaterials, Laser Photon. Rev. 12, 1700220 (2018)

2. A. Krasnok, A. Alú, Embedded scattering eigenstates using resonant metasurfaces, J. Opt. 20, 064002 (2018)

3. D. Ramaccia, F. Bilotti, A. Toscano, Angular momentumbiased metamaterials for filtering waveguide components and antennas with non-reciprocal behavior, in 2014 8th International Congress on Advanced Electromagnetic Materials in Microwaves and Optics (IEEE, 2014), pp. 250-252

4. D. Ramaccia, A. Toscano, F. Bilotti, Scattering and absorption from super-spherical nanoparticles: analysis and design for transparent displays [Invited], J. Opt. Soc. Am. B 34, D62 (2017)

5. D. Ramaccia, S. Arcieri, A. Toscano, F. Bilotti, Core-Shell Super-Spherical Nanoparticles for LSPR-Based Sensing Platforms, IEEE J. Sel. Top. Quantum Electron. 23, 380 (2017)

6. M.G. Silveirinha, Trapping light in open plasmonic nanostructures, Phys. Rev. A 89, 023813 (2014)

7. S. Lannebrio, G. Silveirinha, Optical meta-atom for localization of light with quantized energy, Nat. Commun. 6, 8766 (2015)

8. T. Nakanishi, T. Otani, Y. Tamayama, M. Kitano, Storage of electromagnetic waves in a metamaterial that mimics electromagnetically induced transparency, RAPID Commun. Phys. Rev. B 87, 161110 (2013)

9. C.W. Hsu, B. Zhen, J. Lee, S.-L. Chua, S.G. Johnson, J.D. Joannopoulos, M. Soljačic, S. Soljačić1, Observation of trapped light within the radiation continuum, Nature 499, 188 (2013)

10. Z. Sakotic, A. Krasnok, N. Cselyuszka, N. Jankovic, A. Alú, Berreman embedded eigenstates for narrow-band absorption and thermal emission, Phys. Rev. Appl. 10, 64073 (2020)

11. S. Hrabar, B. Jelacic, L. Mandic, J. Papak, Towards experimental verification of zero-reflection from time-varying capacitor, in 2019 13th International Congress on Artificial Materials for Novel Wave Phenomena, Metamaterials 2019 (Institute of Electrical and Electronics Engineers Inc., 2019), pp. X164-X165

12. D. Ramaccia, D.L. Sounas, A. Alu, A. Toscano, F. Bilotti, Phase-induced frequency conversion and doppler effect with time-modulated metasurfaces, IEEE Trans. Antennas Propag. 68, 1607 (2020)

13. D. Ramaccia, D.L. Sounas, A. Marini, A. Toscano, F. Bilotti, Electromagnetic isolation induced by time-varying metasurfaces: non-reciprocal bragg grating, IEEE Antennas Wirel. Propag. Lett. 19, 1886 (2020)

14. M.S. Mirmoosa, G.A. Ptitcyn, V.S. Asadchy, S.A. Tretyakov, Time-varying reactive elements for extreme accumulation of electromagnetic energy, Phys. Rev. Appl. 11, 014024 (2019)
15. G.A. Ptitcyn, M.S. Mirmoosa, V.S. Asadchy, S.A. Tretyakov, Time-modulated reactive elements for control of electromagnetic energy, in 2019 URSI International Symposium on Electromagnetic Theory, EMTS 2019 (Institute of Electrical and Electronics Engineers Inc., 2019)

16. F.S. Cuesta, V.S. Asadchy, A.D. Sayanskiy, V.A. Lenets, M. S. Mirmoosa, X. Ma, S.B. Glybovski, S.A. Tretyakov, Nonscattering metasurface-bound cavities for field localization, enhancement, and suppression, IEEE Trans. Antennas Propag. 68, 1689 (2019)

17. V.S. Asadchy, M. Albooyeh, S.N. Tcvetkova, A. Díaz-Rubio, Y. Ra'Di, S.A. Tretyakov, Perfect control of reflection and refraction using spatially dispersive metasurfaces, Phys. Rev. B 94, 075142 (2016)

18. S. Taravati, Giant linear nonreciprocity, zero reflection, and zero band gap in equilibrated space-time-varying media, Phys. Rev. Appl. 9, 064012 (2018)

19. F. Monticone, A. Alù, Embedded photonic eigenvalues in $3 \mathrm{D}$ nanostructures, Phys. Rev. Lett. 112, 213903 (2014)

20. D.G. Baranov, A. Krasnok, A. Alù, Coherent virtual absorption based on complex zero excitation for ideal light capturing, Optica 4, 1457 (2017)

21. A. Krasnok, D. Baranov, H. Li, M.-A. Miri, F. Monticone, A. Alú, Anomalies in light scattering, Adv. Opt. Photonics 11, 892 (2019)

22. H. Li, A. Mekawy, A. Krasnok, A. Alú, Virtual parity-time symmetry, Phys. Rev. Lett. 124, 193901 (1939)

23. G. Trainiti, Y. Ra'di, M. Ruzzene, A. Alú, Coherent virtual absorption of elastodynamic waves, Sci. Adv. 5, eaaw3255 (2019)

24. Y. Ra'di, A. Krasnok, A. Alù, Virtual critical coupling, ACS Photonics 7, 1468 (2020)

25. A.V. Marini, D. Ramaccia, A. Toscano, F. Bilotti, Metasurface-bounded open cavities supporting virtual absorption: free-space energy accumulation in lossless systems, Opt. Lett. 45, 3147 (2020)

26. A. Marini, D. Ramaccia, Metasurface-bounded open cavities supporting virtual absorption: Free-space energy accumulation in lossless systems, in 2019 13th International Congress on Artificial Materials for Novel Wave Phenomena, Metamaterials 2019 (Institute of Electrical and Electronics Engineers Inc., 2019), pp. X242-X244

27. A. Marini, D. Ramaccia, A. Toscano, F. Bilotti, Perfect matching of reactive-loaded transmission lines through complex excitation, in 14th European Conference on Antennas and Propagation, EuCAP 2020 (Institute of Electrical and Electronics Engineers Inc., 2020)

28. Advanced Design System (ADS) Simulation Elements Software|Keysight (formerly Agilent's Electronic Measurement), https://www.keysight.com/en/pc-1375582/advanced-designsystem-ads-simulation-elements?cc $=\mathrm{US \& l}=\mathrm{eng}$

29. D.M. Pozar, Microwave engineering (Wiley, 2012)

30. S. Lepeshov, A. Krasnok, Virtual optical pulling force, Optica 7, 1024 (2020)

Cite this article as: Angelica Viola Marini, Davide Ramaccia, Alessandro Toscano, Filiberto Bilotti, Metasurface virtual absorbers: unveiling operative conditions through equivalent lumped circuit model, EPJ Appl. Metamat. 8, 3 (2021) 